\title{
Regulation of root architecture by Pseudomonas oryzihabitans is mediated by strigolactones and redox processes.
}

\author{
Daniel Cantabella ${ }^{1}$, Barbara Karpinska ${ }^{2}$, Neus Teixido ${ }^{3}$, Ramon Dolcet-Sanjuan ${ }^{3}$, and \\ Christine Foyer ${ }^{1}$ \\ ${ }^{1}$ University of Birmingham Edgbaston Campus \\ ${ }^{2}$ University of Birmingham \\ ${ }^{3}$ IRTA
}

August 23, 2020

\begin{abstract}
Mechanisms that control of root system architecture are well characterised but little is known about how these processes respond to plant growth promoting rhizobacteria. We therefore studied how the presence of Pseudomonas oryzihabitans PGP01 altered wild type RSA and how these changes were modified in mutants that are defective antioxidant capacity (vtc2-1, vtc2-2, pad2-1, cad2-1 and rax1-1) or strigolactone (SL) synthesis (max3-9 and max4-1) or signalling (max2-3). The presence of P. oryzihabitans PGP01 decreased the length of primary and lateral roots but increased the number of lateral roots and lateral root density in the wild type roots but not in the SL mutants. The presence of synthetic SL, GR24 in combination with P. oryzihabitans PGP01 significantly decreased the number and length of lateral roots in the WT, max3-9 and max4-1 but not max2-3 seedlings. Lateral root density was increased in all genotypes in the presence of bacterium, but this effect was less pronounced in the ascorbate deficient vtc2-1 and vtc2-2 roots and absent from glutathione -deficient (pad2-1, cad2-1 and rax1-1) seedlings. Taken together, these results demonstrate the importance of SL-mediated signalling in root responses to growth promoting rhizobacteria, as well roles of cellular redox controls in these processes.
\end{abstract}

Pseudomonas oryzihabitans,

\section{Acknowledgements}

The authors would like to express their gratitude to the CERCA Programme/Generalitat de Catalunya, IRTA for the funding of the present study. We also thank the government of Catalonia and the European Social Fund (ESF) "ESF invest in your future" for the Ph.D grant 2018FI_B00641 (Cantabella, D.).

\section{Summary statement}

The presence of the plant growth promoting rhizobacterium,Pseudomonas oryzihabitans PGP01 alters root system architecture in Arabidopsis thaliana in a strigolactone-and glutathione-dependent manner.

\section{Introduction.}

Root system architecture (RSA), which is the term used to describe the organization of the primary and lateral roots (and any accessory roots and root hairs on a microscale), is a key determinant of nutrientand water-use efficiency in plants. Root architecture is defined by the distribution and size of the primary root, adventitious roots, as well as the arrangement of secondary and tertiary roots (Shekhar et al. 2019). The first root structure that appears upon germination is the radicle, from which the primary root develops. Taproots are characterized by the dominance of the primary roots after germination and from which secondary lateral roots develop. This class of root system, also known as allorhizic, is found in the model 
plant Arabidopsis thaliana. In contrast, fibrous or homorhizic root systems are found in monocotyledonous species, where post-embryonic secondary roots that develop adventitious roots, dominate root system architecture after germination. The molecular control of root branching that facilitates the elaboration of root system architecture is essential for the adaptation of the plant to the nutrients and microbiome of the local soil environment. While the regulation of RSA by phytohormones and nutrients is relatively well characterised, much less information is available on how soil microorganisms shape the root system. A. thaliana is frequently used as model for studies on root system architecture (Kellermeier et al. 2014) largely because of the simplicity of the root system, and the broad range of molecular tools that have been developed for this species (Shekharet al. 2019). However, the genetic networks that control RSA in response to the microbiome remain largely unclear.

The successful detection and uptake of nutrients from the soil depends on the capacity of post-embryonic organogenesis and the plasticity of the primary root (PR). Lateral roots (LR) are formed from xylem pools pericycle (XPP) founder cells on the PR. Eight distinctive stages of LR development have been described. In the early stages (I to IV) XPP cells dedifferentiate and proliferate within the endodermis to form the lateral root primordia (LRP), which continually divide and grow through the cellular layers from the endodermis, emerging only at the latest stages of development (Péret et al. 2009). Pre-branch sites in which cells acquire competence to develop LRs are marked by an auxin-dependent signal in the transition zone between proliferating and elongating cells of the PR meristem (Möller et al. 2017). Both the initiation and development of LRs are complex processes that are controlled by a range of molecular pathways. For example, reactive oxygen species (ROS) play a crucial role in LR formation (Manzanoet al. 2014). Genetic manipulation of ROS levels increases the number of pre-branch sites and facilitates the emergence of LR primordia (OrmanLigeza et al. 2016). Other redox-active compounds such as the glutathione ( $\gamma$-glutamyl-cysteinyl-glycine; GSH), thioredoxins (TRX) and glutaredoxins (GRX) are important in the control of root development. Soil microorganisms, such as plant growth promoting rhizobacteria (PGPR) also modulate root development and RSA. For example, PGPR increase LR density in a salicylic acid (SA)-dependent manner and stimulate root hair (RH) elongation (Poitout et al.2017).

Many microorganisms inhabit the rhizosphere and establish communication with the plant roots. It has been estimated that $10^{6}-10^{9}$ bacteria, and $10^{5}-10^{6}$ fungi per gram of soil compete for carbon-based metabolites derived from the root (Chuberreet al. 2018). However, the effect of microorganisms on the root system depends on the species that establishes communication and interactions with the root. Some interactions are beneficial to the plants and others are harmful. Pathogenic soil microorganisms such asFusarium oxysporum and Ralstonia solanacearum cause severe soil borne-diseases in important crop species (Haas \& Défago 2005). Soil-borne diseases can be controlled by the microbiome status of the soil in what is known as diseasesuppressive soil effects, which largely rely on competition between species of microorganisms for the uptake of plant nutrients from the rhizosphere (Schlatter et al. 2017). Conversely, PGPR, which are comprised of different orders of bacterial species, can establish mutualistic interactions with the plants in the rhizosphere, which positively affects the capacity of the root to explore the soil and uptake nutrients (Glick 2012). The mechanisms by which PGPR induce positive changes in the root system remain poorly characterised, but they are considered to rely on either the modification of nutrients sources in the rhizosphere or by direct or indirect effects on phytohormones (Poitout et al. 2017). Bacteria such as Rhizobium spp. secrete nitrogenases that improve the fixation of nitrogen in anaerobic soils, as well as releasing organic acids to increase plant uptake of phosphorus (Yanni et al. 2001). They also induce changes in RSA by altering the levels of ethylene through the synthesis of rhizobitoxine, which inhibits the activity of 1-aminocyclopropane-1-carboxylate synthase and thus decreases the synthesis of ethylene (Yuhashi et al. 2000). Some PGPR species such as Pseudomonas aeruginosa, Klebsiella spp., Rhizobium spp., Mesorhizobium spp., also have the capacity to secrete phytohormones that alter RSA, such as the auxin indole-3-acetic acid (IAA), which is a major player in the control of the root system (Ahemad \& Kibret 2014).

Low molecular weight antioxidants, such as ascorbate (ASC) and GSH are involved in the regulation of signalling processes, which affect plant growth and development (Kocsy et al. 2013). The characterisation of A. thaliana mutants that have low levels of GSH and ASC has impacted these antioxidants in the control of 
root development. While the GSH-deficient root meristemless 1 cannot generate a root system, other weak mutants in the GSH1 gene that accumulate 20-30\% wild-type GSH are able to produce roots. For example, the phytoalexin-deficient2 (pad2-1), the cadmium-sensitive (cad2-1) and rax1-1 show little impairment of the root system (Cobbett et al. 1998; Parisy et al. 2007; Schnaubelt et al. 2013). The ASC-deficient vitamin C2 (vtc2) mutants show altered growth that is linked to changes in the expression of genes encoding proteins involved in phytohormone pathways, particularly auxin, cytokinins, abscisic acid, brassinosterioids, ethylene and salicylic acid (Caviglia et al.2018). The vtc1 mutants contain between 30-60\% of the ascorbate present in wild-type plants and it showed enhanced sensitivity to drought stress (Niu et al. 2013). The Gossypium hirsutum(Gh)VTC1 gene was reported to be a positive regulator of root cell elongation in $A$. thaliana mutants, a process that was under the control of ethylene (Song et al. 2019). However, it is not known how ROS and antioxidants such as GSH and ASC influence root responses to PGPR. In this study, the responses of RSA to P. oryzihabitansstrain PGP01 were compared in the wild type and mutants that are deficient in either strigolactone (SL) synthesis (More Axillary Growth $\{\max \}$ 3-9 and $\operatorname{max4}$-1 ) or SL signalling (max23 ) to test the hypothesis that SL-dependent pathways are important in plant responses to PGPR. SLs play a key role in RSA through the inhibition of branching (Kapulnik et al. 2011; Rasmussen et al. 2012). They interact with other phytohormone, particularly auxins, to control root morphology (Agusti et al. 2011; Ruyter-Spira et al.2011; Jong et al. 2014). They are also important signalling molecules in plant-microbe interactions attracting arbuscular mycorrhizal fungi or favouring the nodulation between bacteriaRhizobium spp. with legumes (López-Ráez et al. 2017). Secondly, we compared the root responses of the wild type $A$. thaliana, the ASC-deficient vtc2-1 and vtc2-2 mutants and the GSH-deficient pad2-1, cad2-1 and rax1-1 mutants toPseudomonas oryzihabitans strain PGP01. These studies show that plant responses to PGPR are either decreased or absent from the SL mutants and the ASC-deficient and GSH-deficient mutants relative to the wild type.

\section{Material and methods.}

Growth on plates.

Seeds of the A. thaliana Columbia-0 (Col-0) wild-type (WT), the SL-deficient mutants (max2-3 , max3-9 and max 4-1 ), the ASC-deficient (vtc2-1 and vtc2-2) mutants and the GSH-deficient (pad2-1, cad2-1 and rax1-1 ) mutants were surface sterilized for $5 \mathrm{~min}$ in $50 \%$ bleach, followed by three 5 -min-washes with sterilized water. Sterile seeds were then cultured in $10 \mathrm{~cm}$ square petri dishes containing half-strength Murashige and Skoog medium ( $\frac{1}{2}$ MS, pH 5.7), supplemented with $0.01 \%$ myo-inositol, $0.05 \%$ MES, $1 \%$ sucrose and $1 \%$ plant agar. Plates were stored at $4^{\mathrm{O}} \mathrm{C}$ in a dark room between $2-4$ days to synchronise germination, and then plated vertically in a plant growth cabinet with a 16 -h photoperiod and $22^{\circ} \mathrm{C}$ of temperature during 6 days. In order to test the combined effect of the synthetic SL, GR24, and bacteria, 6-day-old Col-0, max2-3, max3-9 and max4-1 seedlings were transferred to new plates containing the same medium plus GR24 (2 $\mu \mathrm{M})$ prior the inoculation with bacteria. For each experiment, at least three plates per treatment and genotype and 6 seeds per plate were used.

Inoculation of bacteria.

Pseudomonas oryzihabitans strain PGP01 was obtained from the IRTA Postharvest Plant Growth Promoter Microorganism (PGPM) Collection (Lleida, Catalonia, Spain). The bacterium were grown in nutrient yeast dextrose agar (NYDA: nutrient broth, $8 \mathrm{~g} / \mathrm{L}$; yeast extract, $5 \mathrm{~g} / \mathrm{L}$; dextrose, $10 \mathrm{~g} / \mathrm{L}$; and agar, $20 \mathrm{~g} / \mathrm{L}$ ) medium for $48 \mathrm{~h}$. The application of the bacterium to Arabidopsis seedlings was conducted following Zamioudis et al. (2013) protocol with some modifications. Bacterial cells were collected from plates in $10 \mathrm{mM} \mathrm{MgSO}_{4}$, and washed by centrifugation at $5000 \mathrm{~g}$ during $5 \mathrm{~min}$. After resuspension in $10 \mathrm{mM} \mathrm{MgSO}_{4}$, bacterium concentration was adjusted to $1 \times 10^{6}$ and $1 \times 10^{7} \mathrm{CFU} / \mathrm{ml}$ by measuring turbidity at $600 \mathrm{~nm}$. Fifty $\mu \mathrm{l}$ of both bacterial inocula were applied at 5 -cm of root tip of 6-days-old Arabidopsis Col-0 seedlings to test their effects in root architecture. For experiments involving mutant's seedlings, only $1 \times 10^{6} \mathrm{CFU} / \mathrm{ml}$ was used.

Root architecture.

After 7 days of co-culture with bacterium, pictures of control and bacteria-treated plates were taken, and 
different parameters such as primary root length, number of visible lateral roots and length of lateral roots were measured using ImageJ software. Lateral root density was calculated by dividing the number of lateral roots by the primary root length for each root analysed.

\section{Statistical analysis.}

All the experiments were repeated at least three times. Data represent the mean \pm standard error (SE) of the mean. Data from the experiments using Col-0 and bacteria were analysed by one-way ANOVA, and the analysis of data from SLs, ASC and GSH mutants experiments was performed by a two-way ANOVA. Statistical significance was judged at the level $P<0.05$, and the Duncan's post hoc test was used for the means separation when the differences were significant using IBM SPSS statistics 25 program.

\section{Results.}

The presence of $P$. oryzihabitans PGP01 reduced the length of the WT Arabidopsis primary root by about 36 and $28 \%$, at bacterial concentrations of $1 \times 10^{6}$ and $1 \times 10^{7} \mathrm{CFU} / \mathrm{ml}$ respectively, after 7 days of co-culture (Figure 1A, E). Moreover, the seedlings had a significantly higher number of lateral roots at the lower $\left(1 \times 10^{6}\right.$ $\mathrm{CFU} / \mathrm{ml}$ ) but not the higher bacterial concentration (Figure 1B, E). The length of the visible lateral roots was not significantly changed in response to the presence of $P$. oryzihabitans PGP01 at either bacterial concentration (Figure 1C). As a consequence of the decreased primary root length and the higher number of lateral roots, lateral root density was increased in the presence of both concentrations $\left(1 \times 10^{6}\right.$ and $1 \times 10^{7}$ $\mathrm{CFU} / \mathrm{ml})$ of $P$. oryzihabitans PGP01, the effect being greatest at the $1 \times 10^{6} \mathrm{CFU} / \mathrm{ml}$ concentration $(100 \%$ increase compared to the WT) (Figure 1D). Based on these results, we choose to use the $1 \times 10^{6} \mathrm{CFU} / \mathrm{ml}$ concentration in all the following experiments.

Root responses in strigolactone (SL) deficient mutants.

In the absence of bacteria, the max2-3, $\max 3-9$ and $\max 4-1$ seedlings had a shorter primary root than the WT seedlings, with decreases of 32, 18 and 42\%, respectively (Figure 2A, E). However, the number of lateral roots was not greatly changed in the mutants compared to the WT seedlings (Figure 2B, E). Moreover, no significant differences were detected for the length of lateral roots in absence of bacteria in either the WT or max2-3 , $\operatorname{max3-9}$ and $\max 4-1$ seedlings (Figure 2C). Lateral root density was increased by 12, 12 and $25 \%$ in these lines respectively, relative to the wild type but these changes were not significant (Figure 2D). While the presence of $P$. oryzihabitans PGP01 decreased the primary root length in the WT, with a higher number of lateral roots and a greater lateral root density after 7 days of co-culture (Figure 2), these bacterium-induced changes were largely absent from the SLs mutants. Onlymax3-9 showed a significant 20\% reduction in primary root length after the presence of $P$. oryzihabitans PGP01 (Figure 2A).

After 7 days growth in media supplemented with the SL analogue GR24 in combination with P. oryzihabitans PGP01, a significant decrease in primary root length was observed in the mutant seedlings relative to the WT (Figure 3A). The presence of GR24 in combination with P. oryzihabitans PGP01 significantly decreased the number of lateral roots in the WT, max3-9 and $\operatorname{max4-1}$ seedlings but notmax2-3 seedlings (Figure 3B). While the presence of GR24 andP. oryzihabitans PGP01 had no consistent effect on the length of lateral roots in the WT and max2-3 seedlings, the lateral roots were significantly shorter in $\max 3-9$ and $\max 4-1$ seedlings that the WT under these conditions (Figure 3C). Similarly, the lateral root densities of the max3-9 and $\max 4-1$ seedlings were lower than the other genotypes under these conditions (Figure 3D).

\section{Ascorbate-deficient mutants.}

The primary roots were significantly shorter in the vtc2-1 and vtc2-2 mutants than the WT in the absence of bacteria (Figure 4A, E). However, the number of lateral roots, the length of lateral roots and lateral root density were similar in all genotypes (Figure 4B, C, D). The number of lateral roots was significantly increased in the wild type seedlings in the presence of $P$. oryzihabitans PGP01 but this effect was less pronounced in the ASC-deficient mutants (Figure 4B). The presence of P. oryzihabitans PGP01 significantly reduced the length of the lateral roots in the wild type and the ASC-deficient mutants (Figure 4C). Lateral 
root density was increased in all genotypes in the presence of bacteria, but this effect was less pronounced in thevtc2-1 and vtc2-2 seedlings than the WT (Figure 4D).

Glutathione-deficient mutants. There were no differences in the length of the primary roots in the GSH deficient mutants (cad2-1, pad2-1 and rax1-1) and the WT in the absence of the bacterium (Figure 5A, E). However, the length of the primary roots of all genotypes was significantly decreased in the presence of $P$. oryzihabitans PGP01 (Figure 5A). The cad2-1, pad2-1 and rax1-1 mutants had fewer visible lateral roots than the WT seedlings in the absence of bacterium (Figure 5B, E). While the presence of P. oryzihabitans PGP01 led to a significant increase in the number of lateral roots in the WT seedlings, no significant bacterium-induced changes were observed for the GSH deficient mutants (Figure 5B). The length of lateral roots was significantly decreased in the WT seedlings in the presence of $P$. oryzihabitans PGP01 but not in the GSH-deficient mutants (Figure 5C). Only the pad2-1 mutants had a lower lateral root density than the $\mathrm{WT}$ in the absence of $P$. oryzihabitans PGP01 (Figure 5D). Lateral root density was significantly increased in all the genotypes in the presence of bacteria. However, while, this increase was about $83 \%$ in the WT compared to seedlings grown in the absence of bacteria, the bacteria-induced effects on this parameter were less pronounced in the GSH-deficient mutants (Figure 5D).

\section{Discussion.}

SLs regulate many important processes in plants including shoot branching, root growth, secondary growth and tolerance to cold and drought stresses (Foo \& Reid 2013; Quain et al. 2014; Jonget al. 2014; Cooper et al. 2018). They are also important regulators of the Rhizobium -legume symbiosis and other rhizosphere plant-microbe interactions, such as the symbiosis with arbuscular mycorrhizal fungi, particularly when nutrients such as phosphorus and nitrogen are limiting (Aliche et al. 2020). The synthetic SL analogue GR24 stimulating swarming motility of the alfalfa symbiont Sinorhizobium meliloti (Pelaez-Vico et al.,2020). The data presented here show that SL are crucial for the appropriate responses of roots to the plant growth promoting rhizobacterium $P$. oryzihabitans PGP01. The presence of this bacterium resulted in a strong response of the WT $A$. thalianaroot system, leading to a decrease in primary root length and an increased lateral root density, changes that were absent from the SL mutants.

Previous studies have shown that the inoculation of nectarine (Prunus persica cv. Nectarina) embryos with $P$. oryzihabitans $\mathrm{PGP} 01$ induced root modifications that promote plant growth under greenhouse conditions (Cantabella et al. 2020). The effects of bacteria belonging to Pseudomonas genus have been previously studied in A. thaliana . For example, a strain of $P$. fluorescens was able to promote the growth of $A$. thaliana seedlings and increased the number of lateral roots (Zamioudiset al. 2013). A similar effect was observed by Ortiz-Castroet al. (2020) using P. fluorescens and P. putida, although the influence of P. oryzihabitans in A. thalianaroot system has not been previously studied. The data presented here clearly implicate SL in $A$. thaliana root responses to $P$. oryzihabitans PGP01. The PGPR P. simiae WCS417r was found to stimulate lateral root formation in $A$. thaliana, a response that was partly regulated by the production of volatile organic compounds by the bacterium. Although nearly all Arabidopsis accessions examined were found to respond positively to WCS417r, there was a large variation between accessions in terms of the extra number of lateral roots formed, and the effect on primary root length (Wintermans et al. 2016). Cyclodipeptides produced by $P$. putida and $P$. fluorescens cultures were shown to stimulate lateral root and root hair formation through the induction of auxin responses (Ortiz-Castroet al. 2020). Since auxins and SLs closely interact in the regulation of RSA, it is root responses to P. oryzihabitans are absent from mutants that are defective in either SL synthesis or signalling. The changes in the root systems of the SL mutants relative to wild type $A$. thaliana seedlings in the absence of bacteria are consistent with previous reports (Ruyter-Spira et al. 2011). Moreover, the application of GR24 supressed the lateral root primordia in the WT decreasing to the number of lateral roots and lateral root density, as reported previously (Ruyter-Spira et al. 2011). In this study, roots of all genotypes growing in the presence of GR24 together with P. oryzihabitans PGP01 show a decrease in primary root length but there was an increase in lateral root density only in the wild type and not any of the lines that are defective in SL synthesis and signalling. These data are surprising because it would be predicted that GR24 would recover the WT phenotype in the max3-9 and $\max 4-1$ seedlings. 
We have previously shown a link between SL and GSH signalling that was dependent on MAX2 in $A$. thaliana (Marquez-Garcia et al.2014). Redox processes are also important in the control of root growth and development (Kocsy et al. 2013) but little attention has been paid thus far to how ROS-processing molecules such as ASC and GSH contribute to plant responses to PGPR. The effects of P. oryzihabitans PGP01 on lateral root density were less pronounced in thevtc2-1 and vtc2-2 mutants than the wild type. Moreover, the number of lateral roots was significantly increased but the length of the lateral roots was decreased in the wild type seedlings in the presence of P. oryzihabitans PGP01 but not in the cad2-1,pad2-1 and rax1-1 mutants. Taken together, these results suggest that an appropriate cellular redox balance is required to promote appropriate root responses to the bacterium. This result is perhaps not surprising given that mutants lacking glutathione peroxidases (GPX) have an altered root phenotype (Passaia et al.2014) and glutaredoxins such as GRXS8 are considered to be major regulators of RSA (Ehrary et al. 2020). Moreover, GSH is required for indole butyric acid (IBA) conversion to indole acetic acid (IAA), suggesting an important role for GSH-dependent regulation of the auxin pathway in root development (Trujillo-Hernandez et al. 2020).

Taken together, these data show that SL and GSH are key components in the A. thaliana root response to $P$. oryzihabitans PGP01 to A. thaliana. They confirm that the pathways of signalling by SL, auxin and GSH are interlinked in the regulation of root architecture in response to rhizosphere signals. It is tempting to suggest therefore that a bacterium-induced oxidative burst in the plasma membrane of root cells is a primary signal of recognition by the plant that redox changes thereafter trigger phytohormone pathways that mediate appropriate responses in root architecture.

\section{Author contributions}

Daniel Cantabella, Christine H. Foyer, Ramon Dolcet and Neus Teixidó developed the project; Daniel Cantabella, Christine H. Foyer and Barbara Karpinska designed and performed experiments; Daniel Cantabella and Barbara Karpinska measured root parameters; Daniel Cantabella and Christine H. Foyer analysed data; Daniel Cantabella and Christine H. Foyer wrote the article, and all other authors read and contributed to previous versions and approved the final version.

\section{References}

Agusti J., Herold S., Schwarz M., Sanchez P., Ljung K., Dun E.A., Brewer P.B., Beveridge C.A., Sieberer T., Sehr E.M. \& Greb T. (2011) Strigolactone signaling is required for auxin-dependent stimulation of secondary growth in plants. Proceedings of the National Academy of Sciences of the United States of America 108 , 20242-20247.

Ahemad M. \& Kibret M. (2014) Mechanisms and applications of plant growth promoting rhizobacteria: Current perspective. Journal of King Saud University - Science 26 , 1-20.

Aliche E.B., Screpanti C., De Mesmaeker A., Munnik T. \& Bouwmeester H.J. (2020) Science and application of strigolactones. New Phytologist $227,1001-1011$.

Cantabella D., Dolcet-Sanjuan R., Casanovas M., Solsona C., Torres R. \& Teixidó N. (2020) Inoculation of in vitro cultures with rhizosphere microorganisms improve plant development and acclimatization during immature embryo rescue in nectarine and pear breeding programs.Scientia Horticulturae $\mathbf{2 7 3}$, https://doi.org/10.1016/j.scienta.2020.109643.

Caviglia M., Mazorra Morales L.M., Concellón A., Gergoff Grozeff G.E., Wilson M., Foyer C.H. \& Bartoli C.G. (2018) Ethylene signaling triggered by low concentrations of ascorbic acid regulates biomass accumulation in Arabidopsis thaliana. Free Radical Biology and Medicine 122 , 130-136.

Chuberre C., Plancot B., Driouich A., Moore J.P., Bardor M., Gügi B. \& Vicré M. (2018) Plant immunity is compartmentalized and specialized in roots. Frontiers in Plant Science $\mathbf{8 7 1}$.

Cobbett C.S., May M.J., Howden R. \& Rolls B. (1998) The glutathione-deficient, cadmium-sensitive mutant, cad2-1, of Arabidopsis thaliana is deficient in $\gamma$-glutamylcysteine synthetase. Plant Journal 16, 73-78. 
Cooper J.W., Hu Y., Beyyoudh L., Yildiz Dasgan H., Kunert K., Beveridge C.A. \& Foyer C.H. (2018) Strigolactones positively regulate chilling tolerance in pea and in Arabidopsis. Plant Cell and Environment41 , 1298-1310.

Ehrary A., Rosas M., Carpinelli S., Davalos O., Cowling C., Fernandez F. \& Escobar M. (2020) Glutaredoxin AtGRXS8 represses transcriptional and developmental responses to nitrate in Arabidopsis thaliana roots. Plant Direct $\mathbf{4}, 1-12$.

Foo E. \& Reid J.B. (2013) Strigolactones: New Physiological Roles for an Ancient Signal. Journal of Plant Growth Regulation32, 429-442.

Glick B.R. (2012) Plant Growth-Promoting Bacteria : Mechanisms and Applications. 2012.

Haas D. \& Défago G. (2005) Biological control of soil-borne pathogens by fluorescent pseudomonads. Nature Reviews Microbiology3 , 307-319.

Jong M. De, Ongaro V. \& Ljung K. (2014) Auxin and Strigolactone Signaling are Required for Modulation of Arabidopsis Shoot Branching by Nitrogen Supply. Plant Physiology 166 , 384-395.

Kapulnik Y., Delaux P.M., Resnick N., Mayzlish-Gati E., Wininger S., Bhattacharya C., Séjalon-Delmas N., Combier J.P., Bécard G., Belausov E., Beeckman T., Dor E., Hershenhorn J. \& Koltai H. (2011) Strigolactones affect lateral root formation and root-hair elongation in Arabidopsis. Planta 233 , 209-216.

Kellermeier F., Armengaud P., Seditas T.J., Danku J., Salt D.E. \& Amtmann A. (2014) Analysis of the root system architecture of Arabidopsis provides a quantitative readout of crosstalk between nutritional signals. Plant Cell 26 , 1480-1496.

Kocsy G., Tari I., Vanková R., Zechmann B., Gulyás Z., Poór P. \& Galiba G. (2013) Redox control of plant growth and development. Plant Science 211, 77-91.

López-Ráez J.A., Shirasu K. \& Foo E. (2017) Strigolactones in Plant Interactions with Beneficial and Detrimental Organisms: The Yin and Yang. Trends in Plant Science 22, 527-537.

Manzano C., Pallero-Baena M., Casimiro I., De Rybel B., Orman-Ligeza B., Van Isterdael G., Beeckman T., Draye X., Casero P. \& del Pozo J.C. (2014) The emerging role of reactive oxygen species signaling during lateral root development. Plant Physiology 165, 1105-1119.

Marquez-Garcia B., Njo M., Beeckman T., Goormachtig S. \& Foyer C.H. (2014) A new role for glutathione in the regulation of root architecture linked to strigolactones. Plant, Cell and Environment37 , 488-498.

Möller B.K., Ten Hove C.A., Xiang D., Williams N., López L.G., Yoshida S., Smit M., Datla R. \& Weijers D. (2017) Auxin response cell-autonomously controls ground tissue initiation in the early Arabidopsis embryo. Proceedings of the National Academy of Sciences of the United States of America 114, E2533-E2539.

Niu Y., Wang Y., Li P., Zhang F., Liu H. \& Zheng G. (2013) Drought stress induces oxidative stress and the antioxidant defense system in ascorbate-deficient vtc1 mutants of Arabidopsis thaliana. Acta Physiologiae Plantarum 35 , 1189-1200.

Orman-Ligeza B., Parizot B., de Rycke R., Fernandez A., Himschoot E., van Breusegem F., Bennett M.J., Périlleux C., Beeckman T. \& Draye X. (2016) RBOH-mediated ROS production facilitates lateral root emergence in Arabidopsis. Development (Cambridge) 143, 3328-3339.

Ortiz-Castro R., Campos-García J. \& López-Bucio J. (2020) Pseudomonas putida and Pseudomonas fluorescens Influence Arabidopsis Root System Architecture Through an Auxin Response Mediated by Bioactive Cyclodipeptides. Journal of Plant Growth Regulation 39 , 254-265.

Parisy V., Poinssot B., Owsianowski L., Buchala A., Glazebrook J. \& Mauch F. (2007) Identification of PAD2 as a $\gamma$-glutamylcysteine synthetase highlights the importance of glutathione in disease resistance of Arabidopsis. Plant Journal 49, 159-172. 
Passaia G., Queval G., Bai J., Margis-Pinheiro M. \& Foyer C.H. (2014) The effects of redox controls mediated by glutathione peroxidases on root architecture in Arabidopsi. Journal of Experimental Botany65 , 14031413.

Péret B., De Rybel B., Casimiro I., Benková E., Swarup R., Laplaze L., Beeckman T. \& Bennett M.J. (2009) Arabidopsis lateral root development: an emerging story. Trends in Plant Science 14, 399-408.

Poitout A., Martinière A., Kucharczyk B., Queruel N., Silva-Andia J., Mashkoor S., Gamet L., Varoquaux F., Paris N., Sentenac H., Touraine B. \& Desbrosses G. (2017) Local signalling pathways regulate the Arabidopsis root developmental response to Mesorhizobium loti inoculation. Journal of Experimental Botany 68 , 11991211.

Quain M.D., Makgopa M.E., Márquez-García B., Comadira G., Fernandez-Garcia N., Olmos E., Schnaubelt D., Kunert K.J. \& Foyer C.H. (2014) Ectopic phytocystatin expression leads to enhanced drought stress tolerance in soybean (Glycine max) and Arabidopsis thaliana through effects on strigolactone pathways and can also result in improved seed traits. Plant Biotechnology Journal 12, 903-913.

Rasmussen A., Mason M.G., de Cuyper C., Brewer P.B., Herold S., Agusti J., Geelen D., Greb T., Goormachtig S., Beeckman T. \& Beveridge C.A. (2012) Strigolactones suppress adventitious rooting in arabidopsis and pea. Plant Physiology 158, 1976-1987.

Ruyter-Spira C., Kohlen W., Charnikhova T., van Zeijl A., van Bezouwen L., de Ruijter N., Cardoso C., Lopez-Raez J.A., Matusova R., Bours R., Verstappen F. \& Bouwmeester H. (2011) Physiological effects of the synthetic strigolactone analog GR24 on root system architecture in arabidopsis: Another belowground role for strigolactones? Plant Physiology 155, 721-734.

Schlatter D., Kinkel L., Thomashow L., Weller D. \& Paulitz T. (2017) Disease suppressive soils: New insights from the soil microbiome.Phytopathology 107, 1284-1297.

Schnaubelt D., Schulz P., Hannah M.A., Yocgo R.E. \& Foyer C.H. (2013) A phenomics approach to the analysis of the influence of glutathione on leaf area and abiotic stress tolerance in Arabidopsis thaliana.Frontiers in Plant Science $4,1-9$.

Shekhar V., Stöckle D., Thellmann M. \& Vermeer J.E.M. (2019) The role of plant root systems in evolutionary adaptation. Current Topics in Developmental Biology 131, 55-80.

Song W., Wang F., Chen L., Ma R., Zuo X., Cao A., Xie S., Chen X., Jin X. \& Li H. (2019) GhVTC1, the Key Gene for Ascorbate Biosynthesis in Gossypium hirsutum, Involves in Cell Elongation Under Control of Ethylene. Cells 8 .

Trujillo-Hernandez J.A., Bariat L., Enders T.A., Strader L.C., Reichheld J.-P. \& Belin C. (2020) A glutathione-dependent control of the indole butyric acid pathway supports Arabidopsis root system adaptation to phosphate deprivation. Journal of Experimental Botany71 , 4843-4857.

Wintermans P.C.A., Bakker P.A.H.M. \& Pieterse C.M.J. (2016) Natural genetic variation in Arabidopsis for responsiveness to plant growth-promoting rhizobacteria. Plant Molecular Biology90 , 623-634.

Yanni G.Y., Rizk R.Y., An F., An Q., An Z., Bai J., Ban Y., Becker J. \& Berger N. (2001) The benifical plant growth-promoting association of Rhizobum leguminosarum bv. trifolii with rice roots. Aust $J$ Plant Physiol 28, 032014.

Yuhashi K.I., Ichikawa N., Ezura H., Akao S., Minakawa Y., Nukui N., Yasuta T. \& Minamisawa K. (2000) Rhizobitoxine production by Bradyrhizobium elkanii enhances nodulation and competitiveness on Macroptilium atropurpureum. Applied and Environmental Microbiology 66 , 2658-2663.

Zamioudis C., Mastranesti P., Dhonukshe P., Blilou I. \& Pieterse C.M.J. (2013) Unraveling Root Developmental Programs Initiated by BeneficialPseudomonas spp. Bacteria. Plant Physiology 162, 304318. 


\section{FIGURE LEGENDS}

Figure 1.- Effect of the application of two different doses of $P$. oryzihabitans PGP01 $\left(1 \times 10^{6}\right.$ and $1 \times 10^{7} \mathrm{CFU} / \mathrm{ml}$ ) on primary root length (A), number of lateral roots (B), length of lateral roots (C) and lateral root density (D) of A. thaliana Col-0. 6-days-old seedlings were inoculated with this bacterium at $5 \mathrm{~cm}$ of the root tip, and the measurements of the different parameters were measured 7 days after the inoculation. In order to support the results, representative pictures of the seedlings (E) are shown. Data shown mean \pm standard error (SE) of the mean of three independent biological replications. In each graph, different letters indicate significant differences $(P<0.05$; analysis of variance and Duncan's post hoc test).

Figure 2.- Effect of the application of $P$. oryzihabitansPGP01 on primary root length (A), number of lateral roots (B), length of lateral roots (C) and lateral root density (D) of mutants that are defective in SLs (max2-3, $\max 3-9$ and $\max 4-1)$.6-days-old seedlings were inoculated with this bacterium at $5 \mathrm{~cm}$ of the root tip, and the measurements of the different parameters were measured 7 days after the inoculation. In order to support the results, representative pictures of the seedlings (E) are shown. Data shown mean \pm standard error (SE) of the mean of three independent biological replications. In each graph, different letters indicate significant differences $(P<0.05$; analysis of variance and Duncan's post hoc test).

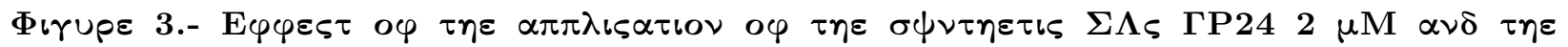

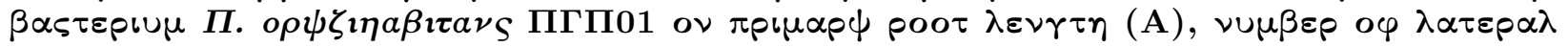

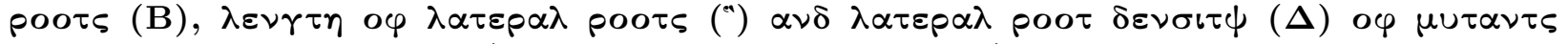

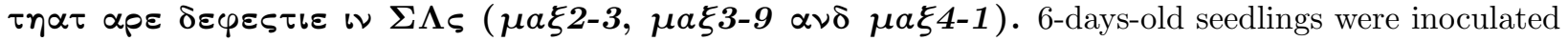
with this bacterium at $5 \mathrm{~cm}$ of the root tip, and the measurements of the different parameters were measured 7 days after the inoculation. In order to support the results, representative pictures of the seedlings (E) are shown. Data shown mean \pm standard error (SE) of the mean of three independent biological replications. In each graph, different letters within the same treatment (Control, GR24 $2 \mu \mathrm{M}$ and GR24 $2 \mu \mathrm{M}+$ PGP01) indicate significant differences $(P<0.05$; analysis of variance and Duncan's post hoc test).

Figure 4.- Effect of the application of $P$. oryzihabitansPGP01 on primary root length (A), number of lateral roots (B), length of lateral roots $(C)$ and lateral root density (D) of mutants that are defective in reduced ASC (vtc2-1 and vtc2-2). 6-days-old seedlings were inoculated with this bacterium at $5 \mathrm{~cm}$ of the root tip, and the measurements of the different parameters were measured 7 days after the inoculation. In order to support the results, representative pictures of the seedlings (E) are shown. Data shown mean \pm standard error (SE) of the mean of three independent biological replications. In each graph, different letters indicate significant differences $(P<0.05$; analysis of variance and Duncan's post hoctest).

Figure 5.- Effect of the application of P. oryzihabitansPGP01 on primary root length (A), number of lateral roots (B), length of lateral roots (C) and lateral root density (D) of mutants that are defective in reduced glutathione (cad2-1, pad2-1 and rax1-1).6-days-old seedlings were inoculated with this bacterium at $5 \mathrm{~cm}$ of the root tip, and the measurements of the different parameters were measured 7 days after the inoculation. In order to support the results, representative pictures of the seedlings (E) are shown. Data shown mean \pm standard error (SE) of the mean of three independent biological replications. In each graph, different letters indicate significant differences $(P<0.05$; analysis of variance and Duncan's post hoc test). 

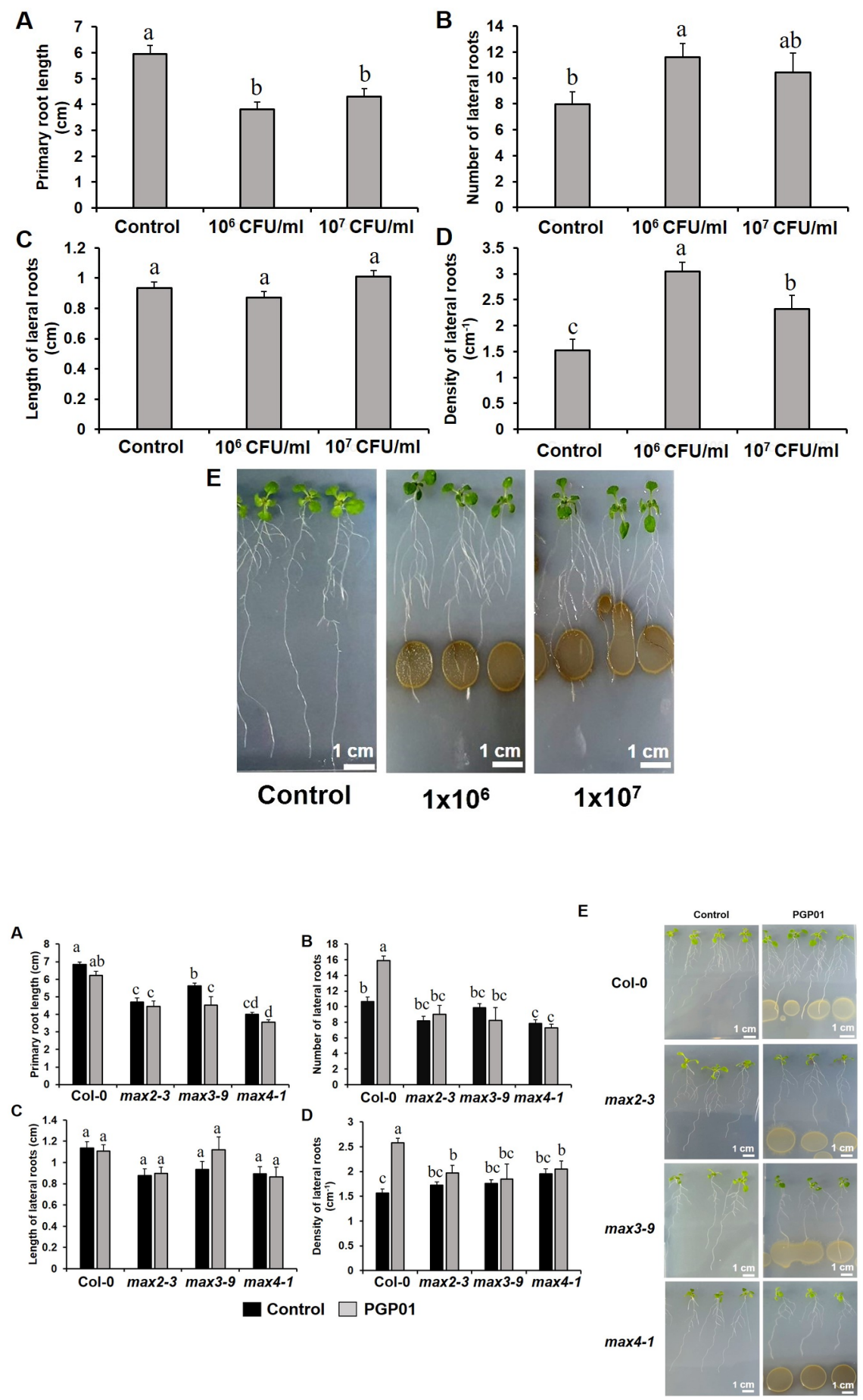

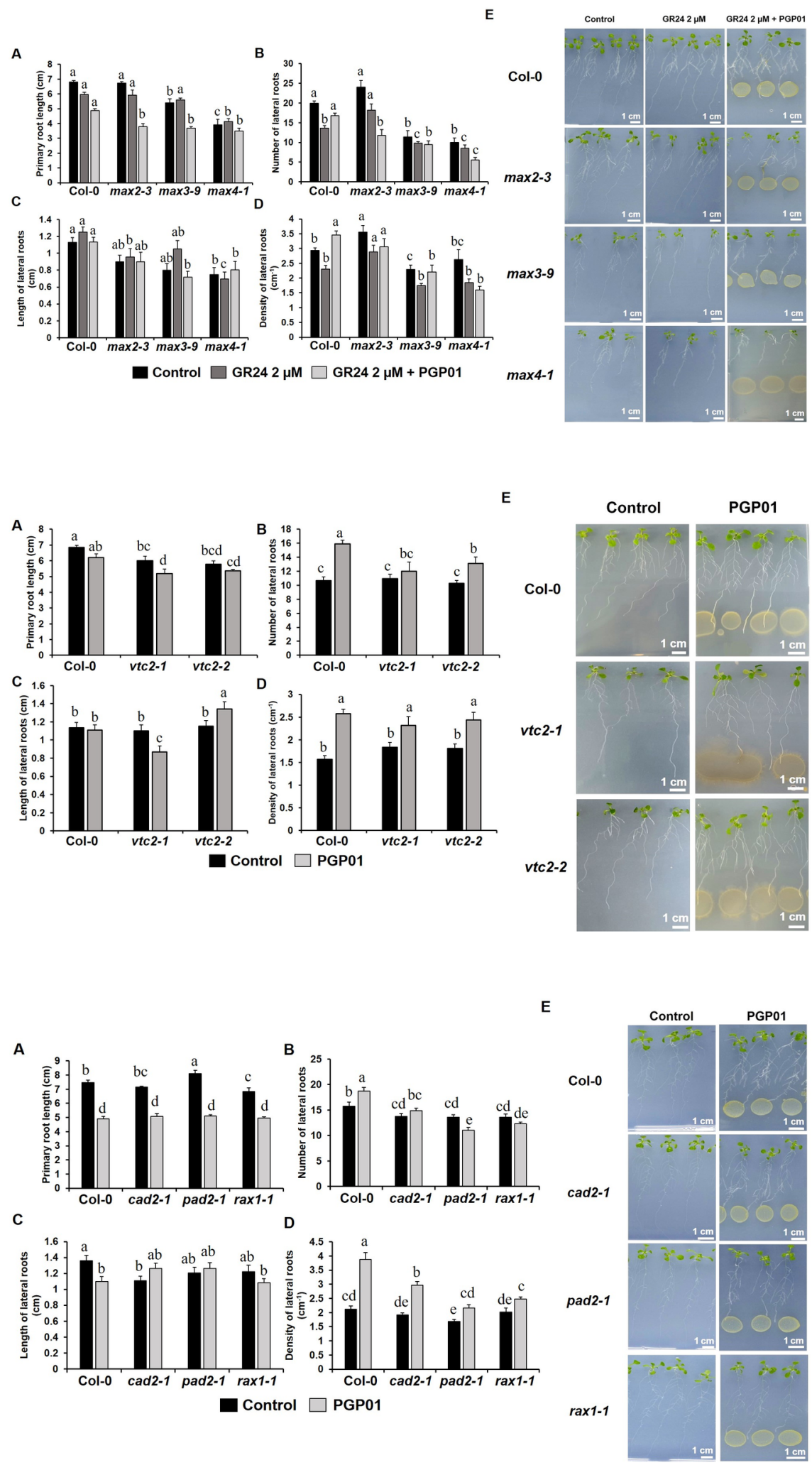